\title{
Les nobles et la ville à la fin du Moyen Âge dans l'espace francophone
}

Vus par les historiens médiévistes

\section{Thierry Dutour}

\section{(2) OpenEdition \\ Journals}

Édition électronique

URL : https://journals.openedition.org/crm/760

DOI : $10.4000 / \mathrm{crm} .760$

ISSN : 1955-2424

\section{Éditeur}

Honoré Champion

Édition imprimée

Date de publication : 30 décembre 2006

Pagination : 151-164

ISSN : 1272-9752

\section{Référence électronique}

Thierry Dutour, "Les nobles et la ville à la fin du Moyen Âge dans l'espace francophone », Cahiers de recherches médiévales [En ligne], 13 | 2006, mis en ligne le 27 novembre 2009, consulté le 15 décembre 2022. URL : http://journals.openedition.org/crm/760 ; DOI : https://doi.org/10.4000/crm.760 


\section{濞RM}

\section{Les nobles et la ville à la fin du Moyen Âge dans l'espace francophone vus par les historiens médiévistes}

La place des nobles dans la société des villes médiévales est un thème de recherche illustré par des travaux variés et nombreux dans les historiographies de langue allemande, anglaise, castillane, flamande, italienne. Tel n'est pas le cas dans l'historiographie de langue française, du moins pour les $\mathrm{XIII}^{\mathrm{e}}-\mathrm{XV}^{\mathrm{e}}$ siècles, sauf à considérer des manifestations d'intérêt très récentes ${ }^{1}$. Ces cheminements contrastés justifient un examen du traitement du thème par l'historiographie de langue française, dans son domaine privilégié : l'espace francophone, espace à la fois linguistique et culturel. La prise en compte de celui-ci présente l'avantage d'éviter l'identification, souvent faite par les historiens français, de l'espace francophone à la France, de celle-ci à la royauté et de cette dernière à l'État. Dans ce cadre, le thème de la place des nobles dans la société des villes n'a guère retenu l'attention. Cela ne résulte pas d'une absence de curiosité mais du sentiment que celle-ci est déjà satisfaite ou n'a pas lieu d'être. Même si la plupart de ceux qui se sont intéressés à la noblesse ont tenu à examiner un thème qui ne leur paraissait pas justifier de très amples développements, il en résulte un désert historiographique dans lequel émerge, à peu près seul, un article de synthèse dû à Philippe Contamine, paru il y a vingt ans ${ }^{2}$. Aujourd'hui encore «l'image traditionnelle de la ville française médiévale est celle d'une ville de bourgeois au milieu d'une campagne dominée par la noblesse des châteaux» (Jacques le Goff) ${ }^{3}$ alors qu'au contraire pour les Temps modernes, sous la plume d'Emmanuel Le Roy Ladurie, la noblesse apparaît urbaine au point qu'on évoque des «cités du sang bleu ${ }^{4}$.

Pourquoi ce désintérêt et avec quelles conséquences? Telle est la question qu'il faut envisager, en sachant qu'embrasser l'ensemble de l'historiographie relative à la noblesse et de celle concernant les villes n'est pas possible, donc sans viser à l'exhaustivité et au risque, évident mais accepté, d'être incomplet et injuste. La constatation de ce que sont les vues présentement exprimées dans l'historiographie française permettra de mettre en valeur la vision de la société qui les sous-tend et les

\footnotetext{
${ }^{1}$ Th. Dutour, (dir.) Les nobles et la vie urbaine dans l'espace francophone XII ${ }^{e}-X V I^{e}$ siècles. Actes de la table-ronde des 17 décembre 2005 et 9 décembre 2006, Paris, Presses de l'université de Paris-Sorbonne, 2007, à paraitre.

${ }^{2} \mathrm{Ph}$. Contamine, «La noblesse et les villes dans la France de la fin du Moyen Âge », Bollettino dell'Istituto storico italiano per il Medio Evo e Archivio Muratiano, 1986, t. 91, p. 467489.

${ }^{3}$ J. Le Goff, «L'apogée de la France urbaine médiévale 1150-1330», dans G. Duby (dir.), Histoire de la France urbaine, t. II, «La ville médiévale des Carolingiens à la Renaissance », Paris, éditions du Seuil, 1980, p. 333.

${ }^{4}$ E. Le Roy Ladurie, dans G. Duby (dir.), Histoire de la France urbaine, t. III, «La France classique de la Renaissance aux révolutions », Paris, Seuil, 1980, p. 391-406. E. Le Roy Ladurie s'inspire notamment de G. Chaussinand-Nogaret, La noblesse au XVIII siècle. De la féodalité aux Lumières, Paris, 1976, réédition Bruxelles, éd. Complexe, 1984.
}

Cahiers de Recherches Médiévales, 13, 2006 
origines, anciennes, de celle-ci. Il sera temps, ensuite, de faire place à des voix dissonantes qui suggèrent d'autres orientations de l'intérêt.

Il est généralement admis que dans l'espace francophone à la fin du Moyen Âge la noblesse est essentiellement, ainsi qu'à la fois, héréditaire, militaire, rurale et fieffée. Elle fait la guerre, vit à la campagne, tient des fiefs. Le mode de vie qui en résulte est suivi par l'écrasante majorité des nobles. Il représente l'idéal de la noblesse. La supériorité sociale à laquelle prétendent les nobles est reconnue par les contemporains et «cette reconnaissance sociale ne saurait être dissociée d'anciennes prérogatives entérinées par le droit» (Martin Aurell) ${ }^{5}$ qui font de la noblesse un ordre et - du moins à partir du XIII ${ }^{\mathrm{e}}$ siècle ${ }^{6}$ - un statut héréditaire. Dans les perspectives les plus courantes, cet état de fait conduit à considérer la place des nobles dans la vie urbaine comme marginale, et à voir dans les nobles citadins une catégorie à peu près négligeable de la population nobiliaire. On avance plusieurs arguments pour justifier cette opinion : des effectifs quantitativement insignifiants, une participation réduite à la vie publique, le fait que les nobles citadins sont une collection d'individualités ne formant pas corps, qu'il s'agit d'ambitieux en quête de réussite sociale cherchant à se glisser dans la noblesse, le fait enfin que si la conception même de la noblesse a fait l'objet de débats parmi les contemporains, ceux-ci restent sans conséquence pratique.

On souligne tout d'abord les effectifs modestes qu'on peut retenir en essayant de recenser des nobles citadins : en règle générale les nobles ne vivent guère en ville, à moins qu'entrent en jeu des circonstances particulières. Martin Aurell énumère celles-ci dans un manuel récent: l'ancienneté de l'urbanisation («en Méditerranée ou en Flandre, maints descendants des chevaliers citadins du $\mathrm{XI}^{\mathrm{e}}$ siècle conservent un palais dans leur quartier d'origine»), le fait qu'une ville soit la capitale d'un prince («les capitales [...] attirent [...] tout un personnel [...] appartenant à la noblesse »), la domination de villes par des nobles («les aristocrates vivent dans les cités dont ils sont les seigneurs»). Mais une accumulation de circonstances ne fait pas une règle, ce dont témoigne le titre même du paragraphe ici cité ( «les magistrats municipaux » $)^{7}$. L'opinion générale est résumée dans l'Histoire de la France urbaine dirigée par Georges Duby, parue en 1980, sous la plume de Jacques Le Goff, dans un paragraphe intitulé «la noblesse et la ville». Le titre lui-même du paragraphe pose, au principe du raisonnement, la distinction de deux entités («la noblesse », «la ville») dont la séparation parait aller de soi. L'auteur y résume une évolution. «En gros, à une France urbaine méridionale où la noblesse habite dans les villes et prend part à la vie municipale s'oppose une France septentrionale où la noblesse est physiquement et politiquement absente». Voilà pourquoi c'est «dans le Midi au moins, parfois au premier rang » que «la noblesse prend part [...] à l'affirmation de la communauté urbaine ». Cependant, il en va tout autrement après $1250:$ «presque partout,

${ }^{5}$ M. Aurell, La noblesse en Occident ( $V^{e}-X V^{e}$ siècles), Paris, Armand Colin, 1996, p. 5.

${ }^{6}$ P. Guilhiermoz, Essai sur l'origine de la noblesse en France au Moyen Âge, Paris, 1902, réimpression Genève, Slatkine, 1979.

${ }^{7}$ M. Aurell, La noblesse..., op. cit., p. 142-143. 
là où il y avait une noblesse urbaine plus ou moins nombreuse, plus ou moins influente, elle cesse de compter dans la ville entre 1250 et 1340 [...] même dans le Midi $»^{8}$. Telle est bien la situation que Bernard Chevalier constate à Tours et fait connaître avec la parution en 1975 de Tours ville royale (1356-1520). Constatant que les nobles sont très peu présents à Tours, Bernard Chevalier en déduit que «dans nos pays et sans doute aussi dans toute la Languedoïl noblesse et vie urbaine s'opposent radicalement $»^{9}$.

Pourquoi ? parce que, conclut l'auteur après un long passage consacré à «Tiers état et noblesse », «la gentillesse est une qualité héréditaire liée à un genre de vie qui exclut le commerce et même la pratique judiciaire ${ }^{10}$. Il partage l'avis de Jacques Le Goff qui, dans l'Histoire de la France urbaine, au paragraphe intitulé «Le problème de la bourgeoisie», souligne, avec force, les limites de tout rapprochement entre bourgeois et nobles, en s'appuyant sur l'exemple de Strasbourg, à la lumière des travaux de Philippe Dollinger ${ }^{11}$ : les mentalités, dominées ici par le souci des affaires, là par celui de la guerre et de la largesse, sont différentes ${ }^{12}$. De ce fait, noblesse et bourgeoisie ne peuvent être confondues. Il y a donc lieu, pour Bernard Chevalier, de traiter à part des autres citadins les nobles, mais aussi les ecclésiastiques. En effet, « les ecclésiastiques physiquement se trouvent bien dans la ville, mais moralement ils sont en dehors, voisins réticents ou alliés bienveillants selon les cas, toujours séparés de la communauté cependant $»^{13}$. La ville est une communauté autant qu'une agglomération d'hommes. On peut, de ce fait, y habiter toute sa vie sans être pleinement un citadin puisque l'on est en dehors de la communauté. Il en va ainsi pour les nobles et c'est une raison de leur absence. Martin Aurell fait écho vingt ans plus tard à Bernard Chevalier : même si «le droit ne permet pas toujours de trancher sur la véritable nature de la noblesse urbaine», il y a lieu de s'interroger sur cette «véritable nature»; la noblesse «ne saurait être, bien sûr, confondue avec la bourgeoisie, statut des élites urbaines jouissant de franchises juridiques et de protection royale $»^{14}$. Voilà pourquoi «l'aristocratie nobiliaire[...] participe de moins en moins à la gestion municipale à partir du XIII ${ }^{\mathrm{e}}$ siècle » selon Albert Rigaudière ${ }^{15}$.

On ne s'étonne donc pas de constater que l'Histoire de la France urbaine, au volume déjà cité, dans sa troisième partie, due à Jacques Rossiaud et consacrée aux années 1330-1530, ne comporte aucun paragraphe consacré aux nobles, mentionnés principalement dans deux développement consacrés respectivement à «Riches et pauvres » et «Citadins et ruraux». On y apprend qu'en diverses villes «la noblesse urbaine se désagrégeait en tant que corps » et qu'y comptait seulement un monde de gens «honorables» rassemblant «l'élite des bourgeois, des robins et des marchands », qui - c'est Bernard Chevalier qui est cité ici par Jacques Rossiaud - «se

${ }^{8}$ J. Le Goff, « L'apogée de la France urbaine médiévale... », op. cit., p. 333-335.

${ }^{9}$ B. Chevalier, La ville de Tours et la société tourangelle 1356-1520, Thèse dact., Université de Paris-Sorbonne, 1972, t. I, p. 195.

${ }^{10}$ B. Chevalier, La ville de Tours..., op. cit., t. II, p. 430.

${ }^{11} \mathrm{Ph}$. Dollinger, «Patriciat noble et patriciat bourgeois à Strasbourg au XIV $\mathrm{X}^{\mathrm{e}}$ siècle », Revue d'Alsace, 1950-1951, p. 52-82.

${ }^{12}$ J. Le Goff, «L'apogée de la France urbaine médiévale... », op. cit., p. 345-347.

${ }^{13}$ B. Chevalier, La ville de Tours..., op. cit., t. I p. 195.

${ }^{14}$ M. Aurell, La noblesse..., op. cit., p. 143.

${ }^{15}$ A. Rigaudière, Gouverner la ville au Moyen Âge, Paris, Economica, 1993, p. 168. 
distinguent des nobles $»^{16}$. C'est bien parce que les nobles en ville ne forment pas corps que Philippe Wolff, au terme d'une étude substantielle consacrée à la noblesse toulousaine, peut affirmer que «au sens rigoureux des termes il n'y a pas eu de noblesse toulousaine $»^{17}$.

Si l'on admet cette vision des choses il est légitime de s'interroger sur l'appartenance réelle à la noblesse des nobles citadins ou, au moins, de souligner la marginalité de leur position dans la noblesse. Les spécialistes de l'histoire de celle-ci qui les prennent en compte n'y manquent pas. Ainsi en est-il, par exemple, de Marie-Thérèse Caron dans sa thèse sur La Noblesse dans le Duché de Bourgogne 13151477, parue en 1987 : les cas qu'elle envisage sont particuliers, tel celui d'une femme noble qui épouse un cordonnier et les milieux nobles des villes du duché de Bourgogne à la fin du Moyen Âge «apparaissent comme des exceptions dans un monde nobiliaire caractérisé essentiellement par sa forte implantation rurale $»^{18}:$ des petits nobles, des nobles de noblesse douteuse, des filles mal mariées par des parents désargentés, des anoblis plus riches de pécune que d'ancêtres. Précisément l'exception attire l'attention car elle peut provoquer la contestation de la qualité nobiliaire du fait de la franchise fiscale attachée, dans le royaume de France, à la qualité de noble suivant les armes et donc susciter des procédures écrites et par là des archives : voilà pourquoi on connaît bien par exemple, grâce à Jean Richard, le cas de Jean Ryolet, noble bourguignon qui eut, en 1455, à prouver sa noblesse ${ }^{19}$. Les nobles citadins, relève-t-on en particulier, ne font pas la guerre et leur genre de vie, par conséquent, n'est pas celui des nobles. Or, «être noble n'était pas une simple décoration : c'était un genre de vie, conditionnant peu ou prou toute l'existence $»^{20}$.

Finalement, l'interrogation sur les rapports entre nobles et villes, quand elle va au-delà de la recension des inclassables et des bizarreries, devient une recherche sur «des individus situés à la périphérie de la noblesse» (Philippe Contamine) ${ }^{21}$ qui elle-même ouvre la voie à deux questionnements. Le plus représenté dans l'historiographie francophone porte sur les voies du renouvellement de la noblesse, empruntées par «toute une frange d'aspirants à la noblesse : gens de guerre, de loi et de savoir, officiers et serviteurs du roi, des princes, voire de plus modestes seigneurs, paysans enrichis, bourgeois et marchands $»^{22}$. Voilà pourquoi on retrouve les nobles à partir de 1450 dans l'Histoire de la France urbaine, quand vient «le temps des 'nouveaux messieurs', possesseurs de terres nobles et de manoirs, de douves et de particules, voulant se glisser, 'même au dernier rang' dans la gentilhommerie, tout en conservant une résidence urbaine», qui sont des «nouveaux venus mala-

\footnotetext{
${ }^{16}$ G. Duby (dir.), Histoire de la France urbaine..., op. cit., t. II, p. 489, 498.

$17 \mathrm{Ph}$. Wolff, "La noblesse toulousaine: essai sur son histoire médiévale, », dans $\mathrm{Ph}$. Contamine (textes réunis par), La noblesse au Moyen Âge XI $-X V^{e}$ siècles. Essais à la mémoire de Robert Boutruche, Paris, P.U.F., 1976, p. 153-174.

${ }^{18}$ M.-T. Caron, La Noblesse dans le Duché de Bourgogne 1315-1477, Lille, 1987, p. 245.

${ }^{19}$ J. Richard, «Les états de service d'un noble bourguignon au temps de Philippe le Bon », Annales de Bourgogne, 1957, 29, p. 113-124.

${ }^{20} \mathrm{Ph}$. Contamine, La noblesse au royaume de France de Philippe le Bel à Louis XII. Essai de synthèse, Paris, P.U.F., 1997, p. 45.

${ }^{21} \mathrm{Ph}$. Contamine, La noblesse..., op. cit., p. 45.

${ }^{22} \mathrm{Ph}$. Contamine, «Introduction», dans $\mathrm{Ph}$. Contamine (textes réunis par), La noblesse au Moyen Âge XI ${ }^{e}-X V^{e}$..., op. cit., p. 34.
} 
droits» (Jacques Rossiaud) ${ }^{23}$. On admet en effet comme relevant de l'évidence une fascination des élites citadines pour les splendeurs nobiliaires qui conduit ces élites à vouloir imiter les nobles - on interprète de cette façon, par exemple, l'organisation en ville de tournois au début du $\mathrm{XIV}^{\mathrm{e}}$ siècle - et à voir dans l'accession à la noblesse l'étape ultime de l'ascension sociale. Celle-ci, in fine, s'accompagne inévitablement d'un mode de vie qui éloigne de la ville et fait de marchands conquérants des hobereaux rentiers. Des travaux bien connus illustrent cette vision des choses : par exemple, l'étude, due à Philippe Wolff et parue en 1942, sur la famille Ysalguier de Toulouse ; à l'origine est un changeur, ensuite viennent des possesseurs de seigneuries $^{24}$. Le second questionnement, illustré en particulier par les travaux de Philippe Contamine, porte sur la conception de la noblesse. L'idéal partagé par les nobles, en tant qu'on peut le saisir, n'est pas toujours suivi dans la pratique. L'existence de nobles citadins est une illustration de cet état de choses. La rapidité, abondamment attestée, du renouvellement des familles nobles, en est une autre : «la fluidité de ce groupe social [...] demeurait plus ou moins dissimulée, ce qui explique peut-être qu'en dépit d'un renouvellement assez rapide [...] les nobles aient eu le sentiment d'appartenir à un corps relativement immuable et en grande partie héréditaire ${ }^{25}$. Ils n'étaient pas aveugles, cependant, et les débats sur la justification de la qualité nobiliaire, dans lesquels sont confrontés des arguments sur l'importance respective de l'hérédité et de la vertu, eurent un écho incontestable chez les nobles francophones de la fin du Moyen Âge. Pour autant, on admet de façon générale, avec Philippe Contamine que «cette controverse [...] demeura largement dans le domaine du normatif. Il s'agissait seulement de rappeler la noblesse à ses devoirs $»^{26}$ et, au bout du compte, la réflexion sur ce thème est «demeurée, dans l'espace culturel et politique français, au niveau de l'implicite $»^{27}$.

Les différents aspects de la vision des choses dont on a essayé de donner un aperçu s'appuient sur des travaux qui, pour beaucoup d'entre eux, font, à juste titre, autorité. N'en prenons qu'un exemple : l'évolution qui conduit à ce retrait des nobles de la vie citadine à partir du XIII ${ }^{\mathrm{e}}$ siècle dont il est admis qu'il s'agit d'une évolution générale. Elle est attestée dès le XII ${ }^{\mathrm{e}}$ siècle souvent, ainsi à Laon $^{28}$ ou dans le Ven-

${ }^{23}$ G. Duby (dir.), Histoire de la France urbaine..., op. cit., t. II, p. 609.

${ }^{24} \mathrm{Ph}$. Wolff, «Une famille du XIII ${ }^{\mathrm{e}}$ au XVI ${ }^{\mathrm{e}}$ siècle : les Ysalguier de Toulouse », Mélanges d'histoire sociale, 1942, p. 35-58.

${ }^{25} \mathrm{Ph}$. Contamine, «Introduction», op. cit., p. 34.

${ }^{26} \mathrm{Ph}$. Contamine, La noblesse au royaume de France..., op. cit., p. 302.

${ }^{27} \mathrm{G}$. et Ph. Contamine, «Noblesse, vertu, lignage et 'anciennes richesses'. Jalons pour l'histoire médiévale de deux citations : Juvénal, Satires 8, 20 et Aristote, Politique 5,1 », dans P. Lardet (éd.), La tradition vive. Mélanges d'histoire des textes en l'honneur de Louis Holtz, Turnhout, Brepols, 2003, p. 321-334.

${ }^{28}$ A. Saint-Denis, Apogée d'une cité : Laon et le Laonnois aux XII et XIII ${ }^{e}$ siècles, Nancy, Presses universitaires de Nancy, 1994. 
dômois ${ }^{29}$, au XIII ${ }^{\mathrm{e}}$ siècle dans bien des villes, ainsi Dijon ${ }^{30}, \mathrm{Reims}^{31}$, ou Paris ${ }^{32}$. Si l'on admet qu'il y a lieu de parler de noblesse urbaine quand on rencontre des nobles citadins nombreux, formant corps, monopolisant l'exercice du pouvoir en ville, il paraît incontestable qu'il n'y en a pas, ou guère, dans l'espace francophone durant la période considérée ici. Il n'y a donc pas lieu de s'ériger en censeur austère et rêche des erreurs d'autrui. Les études historiques évoluent certes par rectification ponctuelle de l'erreur ou découverte de faits nouveaux mais, aussi et surtout, par un déplacement des points de vue fondant le questionnement sur le passé. Les points de vue justifiant la production historique dont on a voulu rendre compte ont permis un considérable essor des études sur la noblesse et sur les nobles; nul n'imagine plus, aujourd'hui, une noblesse en déclin à la fin du Moyen Âge, nostalgique d'un âge d'or chevaleresque évanoui et bousculée par les ambitions bourgeoises et celle des officiers. Ce qu'on soulignera ici, c'est qu'orienter l'intérêt dans certaines directions a, inévitablement, conduit à en délaisser d'autres. Ces points de vue reposent, en effet, sur des propositions dont on considère qu'il faut accepter l'évidence ${ }^{33}$.

La principale repose sur la conviction, immensément répandue, qu'il existe une sorte d'incompatibilité de principe entre ce qui définit le plus profondément les temps médiévaux et la ville. Celle-ci est vue comme un phénomène perturbateur de l'organisation d'une société au sein de laquelle elle croît, mais qu'elle finit par bouleverser : la ville est une révolution politique, sociale, économique. On l'a cru très longtemps, beaucoup le croient toujours, et les plus grands historiens l'ont affirmé. «La ville que [le bourgeois] rêve de construire », écrit Marc Bloch en 1939 dans La société féodale, «sera, dans la société féodale, comme un corps étranger ${ }^{34}$ et précisément il consacre aux citadins 4 pages et demi sur 702 que compte son livre : c'est peu. Ces vues - qui ne favorisent guère la mise en valeur du rôle de centre que les agglomérations urbaines jouent pour un territoire et l'importance du lien entre ville et campagne - sont, pour l'essentiel, un héritage des conceptions du XVIII siècle et, en particulier, de la certitude ancienne que le bourgeois, par nature s'oppose à ce que l'on appelait «la féodalité ». C'est ce qu'ont cru la plupart des historiens du XIX siècle et, en particulier, les plus grands d'entre eux. Quand il est relatif aux villes, leur propos envisage, avant tout, et le plus souvent exclusivement, les bourgeois et, par voie de conséquence, l'essor urbain, qui leur paraît caractériser les $\mathrm{XI}^{\mathrm{e}}-\mathrm{XIII}{ }^{\mathrm{e}}$ siècles. C'est dans cette perspective qu'on a longtemps défini les $\mathrm{X}^{\mathrm{e}}$ et $\mathrm{XI}^{\mathrm{e}}$ siècle comme âge "précommunal" de l'histoire des villes. Dans cette optique on étudie

${ }^{29}$ D. Barthélemy, La société dans le comté de Vendôme de l'an mil au XIV siècle, Paris, Fayard, 1993.

${ }^{30} \mathrm{~J}$. Richard, Les ducs de Bourgogne et la formation du duché du XI au XIV siècle, Paris, 1954, réédition Slatkine, 1990.

${ }^{31}$ P. Desportes, Reims et les Rémois aux XIII et XIV siècles, Paris, Picard, 1979.

${ }^{32}$ B. Bove, Dominer la ville : Prévôts des marchands et échevins parisiens de 1260 à 1350, Paris, C.T.H.S., 2004.

${ }^{33}$ Elles sont discutées de façon plus détaillée dans: Th. Dutour, «La notabilité urbaine vue par les historiens médiévistes francophones aux $\mathrm{XIX}^{\mathrm{e}}$ et $\mathrm{XX}^{\mathrm{e}}$ siècles », dans L. Jean-Marie (dir.) La notabilité urbaine $X^{e}-X V I I I^{e}$ siècles, Caen, à paraître.

${ }^{34}$ M. Bloch, La société féodale, Paris, 1939, réédition 1968, éd. Albin Michel, p. 491. 
surtout le développement des communes ${ }^{35}$. En effet, l'accession de bien des citadins à l'autonomie dans la gestion des affaires de leur ville et la forme prise par cette autonomie avec l'apparition des communes attirent l'attention. On s'intéresse alors aux villes et aux communes, posant une relation entre ces deux termes; le premier venant, chronologiquement, avant le second, la relation est vue surtout comme une évolution: l'essor des villes aboutit logiquement, autant dire nécessairement, à l'apparition des communes. À partir de là, on s'interroge sur les agents du changement. L'interrogation est généralement basée sur la conviction que la bourgeoisie est le principal ; qu'elle incarne les nouveautés et les valeurs de la société industrielle et démocratique, apparue à la fin du XVIII ${ }^{\mathrm{e}}$ siècle et au début du XIX ${ }^{\mathrm{e}}$ siècle; et qu'elle est née au Moyen Âge. «C'est ainsi », écrit Augustin Thierry, «qu'un nouveau sens historique a été donné à ce qu'on appelait, d'un nom trop modeste, l'affranchissement des communes et qu'on a reconnu tous les caractères d'une véritable révolution»dans cet événement ${ }^{36}$. L'appréhension de la bourgeoisie comme ensemble se fonde sur cette conception de l'évolution. On la délimite juridiquement et en même temps sur la base d'activités : le négoce, avant tout, car il paraît caractéristique des villes. Sur ces deux fondements - le droit, des activités - on en élabore un troisième en définissant la bourgeoisie comme catégorie sociale. On souligne que ceux qui jouent en ville un rôle social éminent ont vocation à être dits bourgeois. On considère que c'est le cas, avant tout, des marchands et que le bourgeois par excellence est un marchand. Telle est la conception, par exemple, de François Guizot en 1830 , quand il évoque «cette nation bourgeoise naissante » et voit dans «la féodalité, la royauté et les communes, $\mathrm{du} \mathrm{X}^{\mathrm{e}}$ au $\mathrm{XIV}^{\mathrm{e}}$ siècle», «les trois éléments fondamentaux de la société civile à cette époque ${ }^{37}$, ou d'Augustin Thierry encore, avec ses Lettres sur l'Histoire de France en $1827^{38}$, son Tableau de l'ancienne France municipale, paru comme partie de son Essai sur l'histoire de la formation et des progrès du tiers état en 1853 et dans ses Vues des révolutions d'Angleterre. Il développe dans ces ouvrages la vision de ce qu'il pense être une évolution concernant plusieurs siècles, dans laquelle s'opposent la bourgeoisie et l'aristocratie ${ }^{39}$. Dans cette perspective, les bourgeois forment bien une catégorie sociale. Sur cette base, on en vient à considérer qu'ils sont, à eux seuls, toutes l'élite sociale citadine et plus encore : un groupe et même un milieu, qui ne peut être conçu que comme distinct du milieu noble, voire antagoniste de celui-ci : comme l'écrit Augustin Thierry, «le fond (de la révolution communale) [...] c'est [...] pour toutes les villes [...] un désir plus ou moins violent de substituer aux pouvoirs féodaux une magistrature élective $»$. C'est ainsi qu'on a entendu par noblesse et bourgeoisie des entités irréductibles l'une à

\footnotetext{
${ }^{35}$ Voir par exemple : A. Giry, Histoire de la ville de Saint-Omer et de ses institutions jusqu'au XIV siècle, Paris, F. Vieweg libraire-éditeur, 1877.

${ }^{36}$ A. Thierry, Essai sur l'histoire de la formation et des progrès du tiers état, suivi de deux fragments du recueil de monuments inédits de cette histoire, Paris, 1853, réédition Genève, Megariotis Reprint, p. 237-238.

${ }^{37}$ F. Guizot, Histoire de la civilisation en France depuis la chute de l'Empire romain jusqu'en 1789, Paris, Didier, 1830, t. V, p. 121-249, citations p. 185 et 188.

${ }^{38}$ A. Thierry, Lettres sur l'Histoire de France, Gand, éditions Vassas, 1827.

${ }^{39}$ Voir par exemple, à propos de la guerre civile en Angleterre: A. Thierry, CEuvres complètes, t. VI, Dix ans d'études historiques, dixième édition, Paris, 1840, p. 66.

${ }^{40}$ A. Thierry, Essai..., op. cit., p. 238.
} 
l'autre et par la seconde une catégorie sociale ayant disputé aux nobles l'hégémonie sociale. Dans cette perspective, le mouvement communal est la première scène d'un long combat entre la noblesse et une bourgeoisie promue acteur collectif né au Moyen Âge d'une histoire supposée avoir un sens. La bourgeoisie médiévale aurait été un ferment de dissolution d'une société aristocratique. Quand on a vu les bourgeois placer de l'argent en terres et devenir nobles, on a parlé de «trahison des bourgeois $»^{41}$; et la ville, en somme, se comprend par opposition à la campagne. L'influence de cette vision des choses a été durable. On la retrouve chez Henri Pirenne, qui «a cru reconnaître l'essence de l'activité politique des villes flamandes et brabançonnes du Moyen Âge, précurseur du parlementarisme de son époque, dans leur qualite 'd'anciennes démocraties'» (Walter Prevenier et Marc Boone) ${ }^{42}$. On la retrouve chez Marc Bloch, quand il écrit que les bourgeois des villes « apportaient à l'Europe un élément de vie sociale nouveau, profondément étranger à l'esprit qu'il est permis de dire féodal ${ }^{43}$.

Dès lors, ceux qui dominent la ville doivent être différents de ceux qui dominent la campagne : ils sont les bourgeois, ou les «patriciens». Les activités de ceux qui dominent la ville, s'ils en ont, doivent être spécifiques à celle-ci et, en somme, non-rurales : les bourgeois doivent être des marchands - comme si l'activité de négoce, par le fait d'une espèce d'essence, s'opposait à la vie rurale. On oppose donc un essor urbain marchand et une noblesse rurale. Les bourgeois, écrit encore Marc Bloch, appartiennent à «des sociétés d'un caractère nettement professionnel» et forment « une classe professionnelle ${ }^{44}$. On souligne que les modes de vie citadins et nobles sont incompatibles, on met en valeur les manifestations de cette incompatibilité. On souligne, aussi, que l'ascension sociale peut passer par la bourgeoisie, mais s'accomplit dans l'accès à la noblesse (ou dans des modes de vie censés ressembler à ceux de la noblesse et les imiter par l'inutilité économique que l'on prête à cette dernière). Ainsi l'éminence sociale urbaine se réalise dans le reniement de ce qui la constitue, car le bourgeois qui connaît la réussite suprême devient noble, et, bien sûr, seigneur à la campagne. On est alors conduit à présenter les élites qui tiennent le haut du pavé en ville comme une sorte d'équivalent citadin de la noblesse, une noblesse parallèle, ou seconde, qui tient en ville le rôle que jouent les nobles à la campagne : ce qui fait de la noblesse et de la bourgeoisie (parfois appelée «patriciat» dans cette perspective) des partenaires complémentaires. Cela justifie que les rapports entre nobles et ville soient toujours envisagés sous l'angle de l'interrogation sur l'existence ou non d'une noblesse citadine: la possibilité de son existence est perturbatrice puisque les nobles sont ruraux, donc elle n'existe pas, ou c'est une

\footnotetext{
${ }^{41}$ M. Boone, «La terre, les hommes et les villes. Quelques considérations autour du thème de l'urbanisation des propriétaires terriens », dans : XXX, La ville et la transmission des valeurs culturelles au bas Moyen Âge et aux Temps modernes - Die Städte und die Übertragung von kulturellen Werten im Spätmittelalter und die Neuzeit - Cities and the transmission of Cultural Values in the late Middle Ages and Early Modern Period, Actes - Abhandlungen - Records, Bruxelles, 1996, p. 153-173.

${ }^{42}$ W. Prevenier, M. Boone, «Les villes des Pays-Bas méridionaux au bas Moyen Âge : identité urbaine et solidarités corporatives », dans : Bulletin du crédit communal, 183, 1993, p. 2542, citation p. 25.

${ }^{43}$ M. Bloch, La société..., op. cit., p. 493.

${ }^{44}$ M. Bloch, La société..., op. cit., p. 489.
} 
sorte de pseudo-noblesse, noblesse parallèle, un «patriciat». Et quand on rencontre des individus expressément qualifiés de nobles et de bourgeois - ce qui est assez courant, et Raymond Cazelles l'avait souligné ${ }^{45}$ - on est tenté de faire ce que même un historien aussi parfaitement rigoureux qu'Édouard Perroy a fait, ne serait-ce qu'une fois : affirmer que le document d'archive se trompe. Ainsi, dans une enquête sur une émeute fiscale survenue à Montbrison en 1308, pense-t-il que Jean du Mas, l'un des procureurs de la ville à une assemblée réunie par le roi Philippe IV à Tours, bourgeois, en même temps qualifié domicellus, l'est «abusivement $»^{46}$.

Au bout du compte, les nobles n'ont rien à faire en ville. Quand ils sont citadins, ils ne constituent pas pour autant une noblesse urbaine ; marginaux en ville, ils le sont tout autant dans la noblesse. Et s'ils n'ont rien à faire en ville, alors l'apparition d'une forme de supériorité sociale propres aux villes, avec la bourgeoisie, est un moment essentiel de l'histoire sociale de l'Europe médiévale, celui de l'irruption plus ou moins sensationnelle et renversante d'une élite nouvelle. Alors, comme l'a écrit Fernand Braudel, « la ville est césure, rupture, destin du monde [...] Quand elle renaît en Europe avec le $\mathrm{XI}^{\mathrm{e}}$ siècle, l'ascension de l'étroit continent commence. Qu'elle fleurisse en Italie et c'est la Renaissance $»^{47}$. De telles vues doivent beaucoup à des auteurs comme Augustin Thierry.

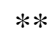

Des voix dissonantes, mais peu entendues et des travaux interprétés d'une façon qui n'était pas la seule possible, incitent depuis longtemps à considérer les choses autrement. Étant donné l'ampleur du sujet, on n'en prendra que quelques exemples.

Celui du raisonnement portant sur les limites de tout rapprochement entre nobles et bourgeois est éclairant. Jacques Le Goff, cité plus haut pour la contribution qu'il y apporte, en s'appuyant sur les travaux de Philippe Dollinger, souligne avec force ces limites ${ }^{48}$. Elles sont incontestables, en effet. Mais le rapprochement l'est autant, à lire Philippe Dollinger, qui parle d'interpénétration des deux mondes. On pourrait mettre l'accent sur le rapprochement. C'est ce que faisait, en 1877, Alfred Giry. Il écrivait, dans son grand ouvrage sur les institutions de Saint-Omer, que «partout en Flandre et en Artois les bourgeois riches, à la fois propriétaires fonciers et commerçants [se sont] unis aux familles féodales qui elles-mêmes faisaient le commerce» au XIII ${ }^{\mathrm{e}}$ siècle. La distinction la plus pertinente lui paraît être celle des gens du commun, exclus des charges publiques, et d'une élite qui rassemble «les riches bourgeois, les grands propriétaires, les gros commerçants, quelques familles

${ }^{45} \mathrm{R}$. Cazelles, La société politique et la crise de la royauté sous Philippe de Valois, Paris, D’Argences, 1958, p. 293-294.

${ }^{46}$ É. Perroy, «Une émeute fiscale à Montbrison sous Philippe le Bel », dans Mélanges offerts à M. Jean Dufour, Montbrison, la Diana, 1940, p. 61-70, citation p. 67.

${ }^{47} \mathrm{~F}$. Braudel, Civilisation matérielle, économie et capitalisme XV $V^{e}$-XVIII ${ }^{e}$ siècle, t. I, «Les structures du quotidien », Paris, Armand Colin, 1979, p. 421.

${ }^{48}$ J. Le Goff, «L'apogée de la France urbaine médiévale... », op. cit., p. 345-347. 
féodales, presque tous formant des lignages échevinaux $»^{49}$. Denis Clauzel souligne lui aussi l'interpénétration en 1996, pour la même région, à propos de Lille. Voulant «poser la question des liens existant entre les élites urbaines et rurales», il constate que «les termes utilisés par les gens du Moyen Âge pour désigner les élites, tant urbaines que rurales, ne diffèrent guère », que «des membres de la société politique communale ont rang de chevaliers ou d'écuyers", que «les similitudes entre les modes de vie» sont fortes et attestées par le droit communal, que «l'espace social urbain témoigne d'une forte emprise nobiliaire » avec «une centaine d'hôtels particuliers » appartenant à des nobles et fort semblables à ceux des membres non-nobles des élites, "que le pouvoir ducal ne dédaigne pas d'utiliser pour loger une partie de la cour $\aleph^{50}$. Si l'on adopte cette perspective, on s'interrogera, non sur la noblesse citadine, mais sur les rapports des nobles et de la ville - ce qui est tout autre chose : car alors on ne raisonne plus sur des ensembles posés a priori, on s'interroge sur des itinéraires et des stratégies individuels et familiaux. Ainsi peut-on s'apercevoir que dans les élites urbaines, on rencontre fréquemment de grandes familles comportant des branches nobles et non-nobles, ainsi à Dijon ou à Paris ${ }^{51}$.

Procéder ainsi conduit donc aussi à envisager autrement, par exemple, ce retrait des nobles hors des villes qui pour beaucoup d'auteurs semble caractéristique des $\mathrm{XII}^{\mathrm{e}}-\mathrm{XIII}^{\mathrm{e}}$ siècles. Il est contemporain de l'évolution qui fait des chevaliers de châteaux des seigneurs de village, connue en particulier par les travaux de Jean Richard $^{52}$ puis de Dominique Barthélemy ${ }^{53}$; on voit mal, à vrai dire, en quoi il s'en distingue. Ce qui frappe alors, ce n'est pas le retrait, sauf à postuler qu'il serait dû à une hypothétique pression des bourgeois dont l'existence est parfois postulée, mais dont la démonstration n'est pas apportée dans l'historiographie existante : c'est qu'il est partiel, qu'à côté de ceux qui partent, il y a ceux qui restent, et c'est l'interpénétration des différentes composantes de l'élite urbaine. Elle avait retenu l'attention d'Arthur Giry pour la Flandre et l'Artois ; on la retrouve en Bourgogne ; on la retrouve à Vendôme, où Dominique Barthélemy n'exclut pas qu' «à défaut de s'éloigner [de la ville, à partir de la seconde moitié du XII ${ }^{\mathrm{e}}$ siècle] certaines lignées chevaleresques ont pu s'agréger ${ }^{54}$ aux autres composantes de l'aristocratie urbaine; on la retrouve à Dijon ${ }^{55}$; on la retrouve encore à Liège $^{56}$ ou à Tarascon ${ }^{57}$;

\footnotetext{
${ }^{49}$ A. Giry, Histoire de la ville de Saint-Omer..., op. cit., p. 160-161. Voir aussi : P. Desportes, Aspects de la Picardie au Moyen Âge, Amiens, C.H.S.U.P., 1995.

${ }^{50} \mathrm{D}$. Clauzel, «Les élites urbaines et le pouvoir municipal : le cas de la bonne ville de Lille aux XIV et XV siècles », Revue du Nord, t. 78, 1996, p. 241-267.

${ }^{51}$ Th. Dutour, Une société de l'honneur. Les notables et leur monde à Dijon à la fin du Moyen Âge, Paris, Honoré Champion, 1998 et B. Bove, Dominer la ville..., op. cit.

${ }^{52}$ J. Richard, "Châteaux, châtelains et vassaux en Bourgogne aux $\mathrm{XI}^{\mathrm{e}}$ et $\mathrm{XII}^{\mathrm{e}}$ siècles », $\mathrm{Ca}$ hiers de civilisation médiévale, 1960, p. 433-447.

${ }^{53}$ D. Barthélemy, La société..., op. cit., p. 749-763.

${ }^{54}$ D. Barthélemy, La société..., op. cit., p. 765.

${ }^{55}$ J. Richard, Les ducs..., op. cit. ; Th. Dutour, «La noblesse dijonnaise dans la seconde moitié du XIV siècle (vers 135O-vers 1410)», dans Ph. Contamine, Th. Dutour, B. Schnerb (textes réunis par), Commerce, finances et société. Recueil de travaux d'histoire médiévale offert à M. le professeur Henri Dubois, Paris, 1993, Presses de l'université de Paris-Sorbonne, p. 311-32 ; Th. Dutour, Une société de l'honneur..., op. cit.

${ }^{56} \mathrm{G}$. Xhayet, Réseaux de pouvoir et solidarités de partis à Liège au Moyen Âge (1250-1468), Genève, Droz, 1997.
} 
enfin, serait-on tenté de dire, dès qu'on est attentif à son existence, on en voit les preuves. Mettre l'accent sur celle-ci, c'est bien déplacer l'intérêt de «la noblesse » vers les nobles. C'est aussi suivre les leçons d'un des meilleurs connaisseurs de l'espace francophone aux $\mathrm{XIII}^{\mathrm{e}}-\mathrm{XV}^{\mathrm{e}}$ siècles : Édouard Perroy, en particulier pour son ouvrage Les familles nobles du Forez au XIII siècle. Essais de filiation, achevé en 1973 et qui fit l'objet d'une publication posthume en 1976. "Toute étude sociale», écrit-il, réside [...] "sur le recensement des familles et l'étude de chacune d'entre elles » qui inclut «tout de qui délimite le niveau social, l'ascension ou le déclin, les fluctuations de la fortune, les protections et les accointances »; et la grande leçon qu'il tire de son étude, c'est, dit-il, «la mobilité sociale ${ }^{58}$.

$\mathrm{Y}$ être attentif permet, par exemple, de s'apercevoir non seulement que le mouvement de retrait de la ville évoqué plus haut est partiel - d'où la nécessité de l'interrogation sur ceux qui ne partent pas, leur devenir, la façon inédite dont ils manifestent une éminence sociale qui prend des formes neuves ne se réduisant pas au fait que ces nobles deviendraient des bourgeois comme les autres ${ }^{59}-$ mais qu'il n'est pas définitif. À d'autres moments on observe le mouvement inverse, connu par exemple grâce aux travaux de Jonathan Dewald ${ }^{60}$, ainsi pour Rouen et son parlement au début du $\mathrm{XVI}^{\mathrm{e}}$ siècle.

Cela conduit aussi à s'interroger sur une des caractéristiques les plus générales des nobles qui est leur attachement à la résidence multiple. Il est mieux connu, sans doute, pour les Temps modernes ${ }^{61}$, mais bien attesté pour ceux qui précèdent quoique mieux mis en valeur quand c'est un moderniste, comme Robert Descimon, qui les envisage ${ }^{62}-$ tant il est vrai que des revenus d'origine rurale ne sont pas incompatibles avec une résidence urbaine ${ }^{63}$. Bien des nobles sont des citadins à temps partiel $^{64}$, ne serait-ce que parce qu'ils passent l'été à la campagne, occupés du souci de leurs propriétés, et l'hiver en ville, tant au Moyen Âge (il n'est que de lire Eustache Deschamps pour s'en convaincre) qu'aux Temps modernes ${ }^{65}$.

${ }^{57}$ M. Hébert, Tarascon au XIV ${ }^{e}$ siècle. Histoire d'une communauté provençale, Aix-enProvence, 1979.

${ }^{58}$ É. Perroy, Les familles nobles du Forez au XIII ${ }^{e}$ siècle. Essais de filiation, Saint-Étienne et Montbrison, Centre d'études foréziennes et la Diana, 1976, t. I, p. 15 et 21. Voir aussi, du même auteur : «Social Mobility among the French 'noblesse' in the later Middle Ages », Past and Present, 1962, p. 25-38.

${ }^{59}$ Thierry Dutour, Une société de l'honneur..., op. cit.

${ }^{60} \mathrm{~J}$. Dewald, Aristocratic Experience and the Origins of Modern Culture: France, 1570-1715, Berkeley, University of California Press, 1993 et du même auteur The European Nobility 1400-1800, Cambridge University Press, 2006.

${ }^{61}$ Cl.-I. Brelot (dir.), Noblesses et villes (1750-1950). Actes du colloque de Tours, 17-19 mars 1994, Tours, Maison des sciences de la ville, 1995.

${ }^{62}$ R. Descimon, «Élites parisiennes entre $X^{\mathrm{e}}$ et XVII ${ }^{\mathrm{e}}$ siècles : du bon usage du Cabinet des titres », Bibliothèque de l'École des Chartes, 1997, 155, p. 607-644.

${ }^{63}$ Un exemple peu connu avec: A. Grimaud, Histoire de Villeneuve-de-Berg, Aubenas, Société de l'imprimerie Habauzit, 1942.

${ }^{64}$ Th. Dutour, La ville médiévale. Origines et triomphe de l'Europe urbaine, Paris, éditions Odile Jacob, 2003.

${ }^{65}$ G. Aubert, «La noblesse et la ville au XVIII ${ }^{\mathrm{e}}$ siècle. Réflexions à partir du cas rennais », Histoire urbaine, $n^{\circ}$ 4, 2001, p. 127-149. 
Cela conduit, pourrait-on ajouter, à s'interroger sur le rôle des villes dans le renouvellement de la noblesse : c'est en ville que s'acquièrent le plus facilement les richesses matérielles ou symboliques - argent, culture et titres académiques, positions de pouvoir, relations - qui n'anoblissent pas mais facilitent l'anoblissement et peuvent, parfois, conduire à le chercher. Les villes et leurs résidents sont une source importante du renouvellement de la noblesse ; Robert Boutruche soulignait qu'en Bordelais les officiers ducaux et les notaires y avaient contribué de façon importante durant la guerre de Cent Ans ${ }^{66}$.

Cela conduit encore à s'interroger sur la participation des nobles à la vie publique en ville : ils ne forment pas corps, c'est vrai : mais cette constatation n'épuise pas la question. On ne décèle aucune incompatibilité entre condition nobiliaire et fonctions municipales, aucune hostilité de principe à la présence de nobles dans les conseils municipaux ou dans les organes par lesquels s'exprime l'existence des collectivités urbaines. Ainsi sur 59 nobles connus à Dijon entre 1350 et 1410, 32 ont été échevin ou maire et les principaux chefs de factions sont des nobles, d'origine pour certains, descendants de familles de chevaliers du XIII ${ }^{\mathrm{e}}$ siècle, anoblis pour d'autres. On trouve une situation comparable à Bordeaux ${ }^{67}$, Chalon-sur-Saône ${ }^{68}$, Lyon $^{69}$, Moulins ${ }^{70}$ et en bien d'autres endroits.

On pourrait allonger cette liste d'interrogations. Elle manifesterait simplement qu'en effet, les voix dissonantes existent depuis longtemps. On n'y prêtait pas attention, et à cet égard il est significatif que l'article de synthèse de Philippe Contamine évoqué plus haut ait eu moins d'écho qu'il l'aurait dû. Sans doute sontelles mieux entendues du fait d'évolutions générales de l'intérêt et des méthodes de travail, qui affectent, plus ou moins rapidement, tous les domaines de spécialité : deux, en particulier, peuvent être mentionnées. La première consiste en un souci de plus en plus manifeste d'envisager les sociétés urbaines pour elles-mêmes - c'est-àdire, non en fonction d'interrogations sur des évolutions pluriséculaires (la naissance du Tiers État, par exemple, ou les différents âges de la féodalité) - qui conduit à ne pas opposer villes et campagne. On remarque alors qu' " en cette fin du Moyen Âge [...] l'attraction des villes [...] se fit sentir avec plus de force que dans la période précédente»(Philippe Contamine) ${ }^{71}$. Dès lors, les relations sociales en ville et la complexité des sociétés urbaines retiennent de plus en plus l'attention. Le souci de la complexité est la seconde inflexion de l'intérêt. S'interroger sur elle, c'est attacher de l'importance à la rencontre et au côtoiement des hommes, créateurs de la réalité sociale qu'ils partagent et considérer les faits sociaux comme des situations, qu'on ne peut comprendre sans s'interroger sur la façon dont les acteurs les définissent ${ }^{72}$.

${ }^{66} \mathrm{R}$. Boutruche, La crise d'une société. Seigneurs et paysans du Bordelais pendant la guerre de Cent Ans, Paris, Les Belles-Lettres, 1963, p. 374.

${ }^{67} \mathrm{R}$. Boutruche, La crise..., op. cit.

${ }^{68} \mathrm{~V}$. Gaillard, Société et économie à Chalon-sur-Saône au XVe siècle, 1989, mémoire pour l'obtention du D.E.A., Université de Paris-Sorbonne.

${ }^{69} \mathrm{R}$. Fédou, Le terrier de Jean Jossard, Paris, 1966.

${ }^{70}$ O. Mattéoni, Servir le prince. Les officiers des ducs de Bourbon à la fin du Moyen Âge (1356-1523), Paris, Publications de la Sorbonne, 1998, p. 132.

${ }^{71} \mathrm{Ph}$. Contamine, «La noblesse et les villes... », op. cit., p. 485.

${ }^{72}$ Th. Dutour, «La réhabilitation de l'acteur social en histoire médiévale», dans : Genèses. Sciences sociales et histoire, ${ }^{\circ}$ 47, 2002, L’individu social, p. 21-41. 
Ainsi apparaissent des façons inédites d'envisager des débats existants. Retenons encore, à titre d'exemple, la question de l'honneur. L'importance attachée à l'honneur est essentielle dans la définition que les écrits des nobles, ou relatifs à la noblesse, proposent de celle-ci. Si l'on admet que nobles et élites citadines nonnobles constituent deux segments irrémédiablement séparés des élites, l'honneur caractérise les nobles. $\mathrm{Si}$, au contraire, on tient que le clivage social essentiel sépare ceux que le vocabulaire du temps appelle - précisément - "gens d'honneur", ou «gens de nom et d'état» ou encore «notables personnes», des autres, désignés au moyen d'un vocabulaire varié et appelés parfois, de façon expressive "gens de néant ${ }^{73}$, on entendra autrement le mot «honneur» et la distinction des nobles et des non-nobles paraîtra certes un clivage social important, mais peut-être pas le plus important, au moins en ce qui concerne les simples gentilshommes. Raymond Cazelles, en 1958, était de cet avis ${ }^{74}$. On admettra, alors, que nobles et bourgeois représentent deux dimensions de la notabilité et, donc, de la supériorité sociale. Les uns et les autres sont des notables, appartiennent à la sanior pars formée de ceux qui ont pleine capacité politique. Ainsi Léopold Genicot, dans sa thèse, parue en 1960, sur L'Économie rurale namuroise au Bas Moyen Âge, relève que les nobles ruraux et les membres des élites citadines «sont également juges, combattants d'élite, personnages riches et hommes honorables ${ }^{75}$ : on pourrait ajouter qu'à la fin du Moyen Âge les nobles sont de plus en plus nombreux à exercer des activités civiles ${ }^{76}$. Dès lors, la place du droit dans la définition de la noblesse sera envisagée autrement: jusqu'au $\mathrm{XVI}^{\mathrm{e}}$ siècle, comme l'a souligné Robert Descimon, "le droit coutumier eut plus d'effet que la législation royale sur les pratiques sociales ${ }^{77}$ et avant les grandes enquêtes de noblesse, on n'est pas noble parce que le roi ou un prince veut bien le reconnaître, mais parce que la société dans laquelle on vit le reconnaît ; on l'est par renommée. Dès lors, le droit issu de la législation royale ou princière, droit écrit, n'a pas forcément vocation à organiser les relations sociales - ce qui veut dire que les sujets du roi et des princes ignorent souvent superbement les ordonnances de ceux-ci et ne s'en portent pas plus mal ; on est, comme l'a joliment dit Jean-Marie Constant, au temps de la noblesse en liberté ${ }^{78}$. C'est alors la notabilité dans son ensemble, en tant qu'en elle s'articulent, d'une façon qui mérite étude, les différentes dimensions de la supériorité sociale («les nobles, bourgeois et autres notables personnes des villes », écrivait Enguerrand de Monstrelet ${ }^{79}$ ), qui sera objet d'examen ${ }^{80}$.

\footnotetext{
${ }^{73} \mathrm{Ph}$. Contamine, La noblesse..., op. cit., p. 313.

${ }^{74}$ R. Cazelles, La société politique..., op. cit., p. 290.

${ }^{75}$ L. Genicot, L'Économie rurale namuroise au Bas Moyen Âge (1199-1429), t. 2, «Les hommes - La noblesse », Louvain, 1960, réimpression anastatique, 1975, p. 288, p. 308-309.

${ }^{76} \mathrm{Th}$. Dutour, «Les nobles, les activités civiles et la vie urbaine dans l'espace francophone (XIIIe-XV ${ }^{\mathrm{e}}$ siècles) », Histoire urbaine, 2006, à paraître.

${ }^{77}$ R. Descimon, «Élites parisiennes... », op. cit., p. 613.

${ }^{78}$ J.-M. Constant, La noblesse en liberté $X V I^{e}-X V I I^{e}$ siècles, Rennes, Presses universitaires de Rennes, 2004

${ }^{79}$ Enguerran de Monstrelet, Chronique, éd. Douët-d'Arcq, Paris, 1860, t. IV, p. 302.

${ }^{80}$ Th. Dutour, «Désigner les notables. Le vocabulaire de la notabilité à la fin du Moyen Âge », dans L. Jean-Marie (dir.), La notabilité urbaine $X^{e}$-XVIII ${ }^{e}$ siècles, Caen, à paraître.
} 


\begin{abstract}
Conclusion
Choisir d'être attentif à la complexité de toute vie sociale c'est admettre qu'une tentative d'explication de cette complexité est, nécessairement, partielle et provisoire et que les conclusions de cette étude le sont aussi. Ont-elles même lieu d'être ? Sans doute pas. Deux constatations en tiendront lieu. Les rapports des nobles et de la ville dans l'espace francophone à la fin du Moyen Âge sont un domaine de recherche mal exploré en dépit des apparences, dans lequel notre savoir présente des lacunes considérables; il serait dommage de ne pas travailler à les combler. Les orientations présentes de la recherche persuadent qu'il y aurait avantage, pour cela, à ne pas postuler que universalia sunt realia ante rem, à considérer comme citadins ceux qui habitent en ville, sans leur refuser cette qualité au motif que leur qualification sociale ou leurs activités les excluent de la population de ceux dont on postule qu'ils sont les «vrais » citadins, enfin à envisager ensemble ce que l'on a longtemps voulu séparer et souvent opposé : villes et campagnes, nobles et bourgeois (et tous les notables qui ne sont ni nobles, ni bourgeois et sont pourtant des notables). Les $\mathrm{XIII}{ }^{\mathrm{e}}-\mathrm{XV}^{\mathrm{e}}$ siècles sont-il marqués, avant tout, par une indépassable distinction entre nobles et bourgeois ? Ou sont-ils marqués par la diversification des dimensions de la supériorité sociale? Les recherches contemporaines suggèrent la pertinence du second terme de l'alternative. Oublions donc Augustin Thierry. Constater que, mort en 1856, il a disparu depuis exactement 150 ans et suggérer qu'on s'émancipe de ses vues n'est pas faire outrage à sa mémoire.
\end{abstract}

Thierry Dutour Université de Paris-Sorbonne 